\title{
The power of teaming up HST and Gaia: the first proper motion measurement of the distant cluster NGC 2419
}

\author{
D. Massari ${ }^{1,2}$, L. Posti ${ }^{2}$, A. Helmi ${ }^{2}$, G. Fiorentino ${ }^{1}$, and E. Tolstoy ${ }^{2}$ \\ 1 INAF-Osservatorio Astronomico di Bologna, via Ranzani 1, 40127 Bologna, Italy \\ e-mail: davide.massari@oabo.inaf.it \\ 2 University of Groningen, Kapteyn Astronomical Institute, 9747 AD Groningen, The Netherlands \\ Received 1 December 2016 / Accepted 25 January 2017
}

\begin{abstract}
Aims. We present the first measurement of the proper motion and orbit of the very distant and intriguing globular cluster NCG 2419. Methods. We have combined data from HST and Gaia DR1 to derive the relative proper motions of stars in the direction of the cluster. To tie these to an absolute reference frame, we have used a background galaxy located in the field.

Results. We find the absolute proper motion of NGC 2419 to be $\left(\mu_{\alpha} \cos (\delta), \mu_{\delta}\right)=(-0.17 \pm 0.26,-0.49 \pm 0.17)$ mas $\mathrm{yr}^{-1}$. We have integrated the orbit of the cluster in a Galactic potential and found it to oscillate between $\sim 53 \mathrm{kpc}$ and $\sim 98 \mathrm{kpc}$ on an almost polar orbit. This, and the fact that it shares the same sense of rotation around the Milky Way, make it very likely that NGC 2419 is a former cluster of the Sagittarius dwarf spheroidal galaxy.
\end{abstract}

Key words. globular clusters: individual: NGC 2419 - astrometry - proper motions

\section{Introduction}

Proper motions (PMs) are an extremely powerful tool for investigating the evolution of the Milky Way and its satellites. In the context of a hierarchical growth of structure, many of the objects (stars, streams, and globular clusters, e.g. Helmi et al. 2017; Belokurov et al. 2006; Dinescu et al. 2002) that we currently observe in the Milky Way halo could have originated outside the Galaxy, brought in by satellites that have survived until the present day or accreted a long time ago. Typically, photometric information alone is not enough to establish such origins robustly. Possibly the most effective way is to determine the orbital trajectories and this requires measurement of their PMs. However, since the size of PMs depends on the distance and on the temporal baseline between the astrometric observations, PMs can be very small and difficult to measure.

Nevertheless, a new golden era for PMs science is rising. The Hubble Space Telescope (HST) has been the most powerful astrometric instrument so far. Thanks to its point spread function (PSF) and geometric distortions being stable within a few percent (Anderson 2007; Bellini et al. 2011), HST has reached astrometric accuracies on single exposures up to $\sim 0.5$ mas for bright stars (Anderson 2007). Very recently, the astrometric satellite Gaia released its first positional measurements (Data Release 1, Gaia Collaboration 2016) for more than 1 billion stars across the whole sky, and a sub-sample of these have positional accuracies comparable to HST. However, these two exceptional instruments have limitations if taken separately. For example, HST often lacks second epoch observations, while at the end of its mission, Gaia will provide PMs obtained on a temporal baseline of only five years. Yet the synergy between HST and Gaia allows us to overcome the main limitations of both. Gaia will provide accurate second epoch measurements for any
HST data, while in several cases, especially for the central regions of crowded stellar systems, HST will provide observations taken up to 15-20 years before Gaia, thus increasing the PMs temporal baseline by a factor of 4-5.

In this work, we describe the first combination of datasets from HST and Gaia to measure the PM and determine the orbit of the enigmatic cluster NGC 2419. NGC 2419 is by far the brightest globular cluster in the outer regions of the Milky Way halo. Its chemistry is comparable to that of massive clusters, with homogeneous $[\mathrm{Fe} / \mathrm{H}]$ measurements (Mucciarelli et al. 2012), and photometric evidence for He enhancement (di Criscienzo et al. 2011a). On the other hand, there are suggestions of a calcium spread (Cohen et al. 2010; Lee et al. 2013), and its halflight radius is significantly larger than that of a typical globular cluster (Harris 1996). This, coupled with its unusually large distance (87.5 kpc, Di Criscienzo et al. 2011b), makes it intriguing. NGC 2419 has been proposed as a candidate nucleus of an accreted dwarf galaxy, and to have been formed inside a dark matter halo (Baumgardt et al. 2009). To shed light on its nature and origin, we present the first estimate of the proper motion of NGC 2419 by exploiting the combination of HST and Gaia. This is then used to compute possible orbits in a realistic Galactic mass distribution. An analysis that is similar in spirit has been performed on five nearby globular clusters by Watkins \& van der Marel (2016) who exploited the Tycho-Gaia solution (TGAS, see Lindegren et al. 2016) rather than using HST. However, since TGAS PMs are available only for bright stars, no such measurements exist for NGC 2419 members.

In Sect. 2 we describe the data analysis and the method used to measure the absolute PM of the cluster. In Sect. 3 we compute the orbit of NGC 2419 and check for possible associations with dwarf galaxies and other known stellar clusters. We summarise our conclusions in Sect. 4. 


\section{Data analysis and PM measurement}

As the first epoch for the PM determination, we used data acquired under GO-9666 (PI: R. Gilliland) with the Wide Field Channel (WFC) of the Advanced Camera for Survey (ACS) on board the HST. The WFC/ACS is made up of two detectors with a size of $2048 \times 4096$ pixels and a pixel scale of $\sim 0.05^{\prime \prime}$ pixel $^{-1}$. Detectors are separated by a gap of approximately 50 pixels, meaning that the total field of view (FoV) is $\sim 200^{\prime \prime} \times 200^{\prime \prime}$. We used 14 deep exposures in the $F 435 W$, $F 475 W, F 555 W, F 606 W, F 625 W, F 775 W$, and $F 814 W$ filters (two exposures per filter), taken on September 26, 2002. We work with_FLC images, which have been corrected by the HST calibration pipeline for charge transfer efficiency (Anderson \& Bedin 2010; and Ubeda \& Anderson 2012). The data reduction is based on the procedures described in Anderson \& King (2006). Each individual exposure was analysed with the publicly available program img $2 x y m \_W F C .09 \times 10$. This program uses a pre-determined model of the PSF plus a single time-dependent perturbation, and produces a catalogue with positions and instrumental magnitudes as output. After rejecting all the saturated sources, the stellar positions in each catalogue were corrected for filter-dependent geometric distortions using the solution provided by Anderson (2007).

The second epoch data are provided by the Gaia Data Release 1 (DR1, see Gaia Collaboration 2016). DR1 positions are from January 1, 2015. In combination with HST, this provides a temporal baseline for the PM measurement of 12.27 years.

A catalogue containing positions, related uncertainties, $G$ magnitudes and astrometric excess noise for all the sources in the FoV covered by the HST dataset was requested from the Gaia archive $^{1}$. We found that the median positional error for this catalogue was $\sim 0.6$ mas, and decided to exclude all the sources with a positional error larger than 3 mas $(\sim 5$ times the median error value) from the analysis to remove poorer quality measurements.

The PMs were measured using the procedure described in Massari et al. (2013). We chose a master frame described by the Gaia positions, which is already aligned with the equatorial coordinate system. Then, we transformed each HST single exposure catalogue onto the master frame using a six-parameter linear transformation. To maximise the accuracy of these transformations, we treated each chip of the HST exposures separately to avoid spurious effects due to the presence of gaps. After this process, each source had up to 14 first-epoch positions transformed onto the master frame. We decided to exclude all those sources with less than four first-epoch detections from the following analysis. The PMs of the remaining 481 objects were computed as the difference between the second epoch Gaia positions and the $3 \sigma$-clipped median value of the HST first-epoch positions, divided by the temporal baseline. The two projected components of the PMs on the sky were treated separately. The uncertainties on the PMs were computed as the sum in quadrature between the Gaia positional errors and the rms of the residuals about the median value of HST positions, divided by the temporal baseline.

After this first iteration, we repeated the procedure by computing the frame transformations using only likely cluster members. We selected stars according to both their location in the colour-magnitude diagram (CMD) and their first PM determination, requiring consistency with the mean cluster motion (which

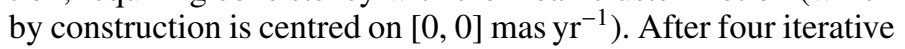

\footnotetext{
1 https://gea.esac.esa.int/archive/
}
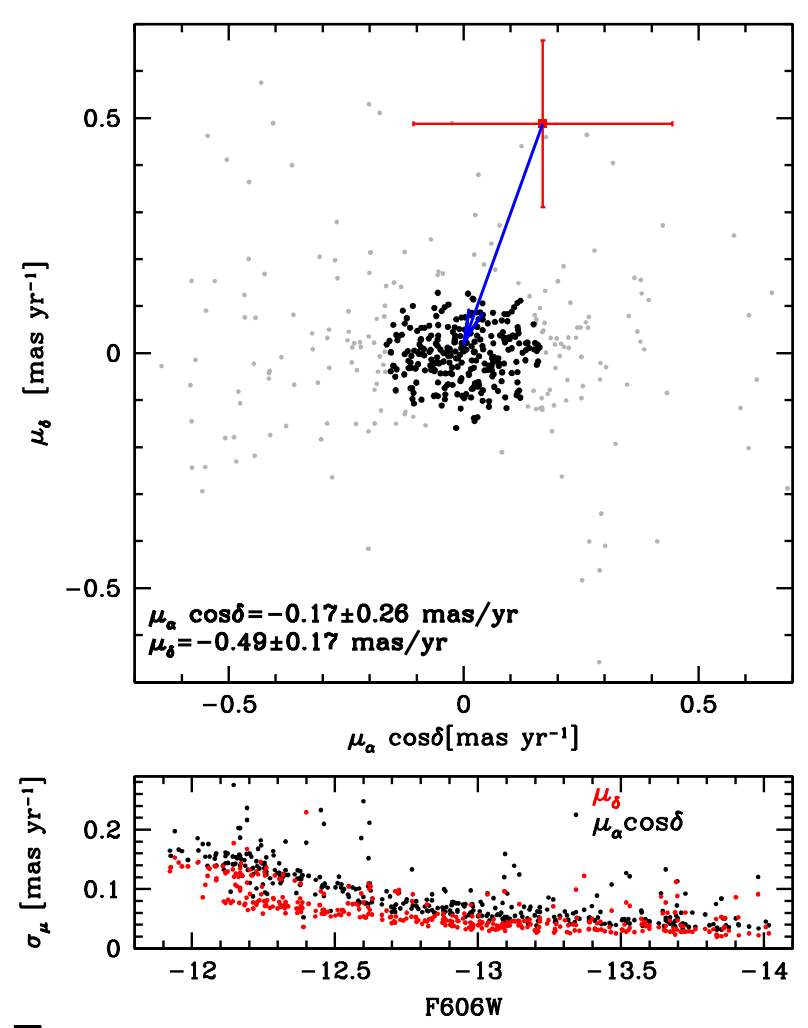

Fig. 1. Top panel: VPD for the stars in our final catalogue. Stars used to measure the average cluster PM are highlighted in black, whereas likely non-members are shown in grey. The location of the background galaxy used to determine the absolute PM zero-point with its uncertainty is shown with a red symbol. Bottom panel: uncertainties on the PM measurements. Black and red symbols are related to the two different PM components as described in the labels.

steps, the selected number of stars ceased to change (366 were used in the last step), and the resulting PMs and related errors are those of our final catalogue. The PMs are shown in the top panel of Fig. 1, also known as a Vector Point Diagram (VPD), while the uncertainties for each PM component are plotted in the bottom panel with different colours. The larger uncertainties on $\mu_{\alpha} \cos (\delta)$ are due to the positional errors in the Gaia dataset, and explain why the distribution of stars in the VPD appears elongated in that direction.

We have performed several consistency checks on these PM measurements. First, we verified that the bulk of the PM distribution centred around zero is actually made up of cluster member stars. We selected stars around the mean PM value in the VPD with an iterative $2.5 \sigma$-clipping procedure. Their location in the instrumental (F606W, F606W-F814W) CMD is shown with black symbols in the left panel of Fig. 2. All the selected stars lie on the cluster evolutionary sequence, and other stars that are also on this sequence (grey symbols) are excluded because of their large PM uncertainties (see Fig. 1). Following Bellini et al. (2014) and Massari et al. (2016), we also checked for spurious systematic trends of the measured PM components with spatial distribution, instrumental magnitude and colour, and found none. This is demonstrated in the top- and bottom-right panels of Fig. 2. The distributions with magnitude and colour are consistent with no systematic trends within a $1 \sigma$ uncertainty. All these checks support the quality and the reliability of our measurements. However, since we are using only two epochs, we cannot exclude that other subtle systematic errors affect our analysis, 

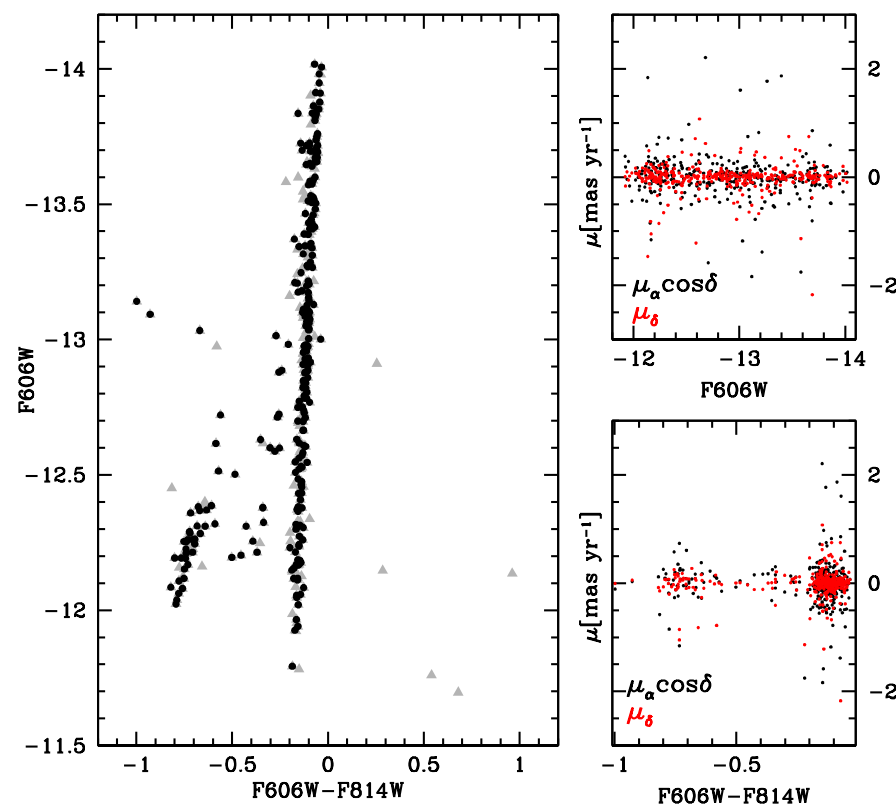

Fig. 2. Left panel: (F606W, F606W-F814W) instrumental CMD of NGC 2419 for the stars in the final PM catalogue. Black and grey symbols indicate stars selected in the VPD as shown in Fig. 1. Right panels: behaviour of the two PM components with respect to instrumental magnitude (top panel) and colour (bottom panel). In both cases, the best fits indicate no systematic trend.

possibly making the overall estimate of the PM uncertainties a lower limit.

The PMs measured in this way are relative to the mean motion of the cluster. Relating them to an absolute reference frame is therefore necessary in order to obtain the systemic motion of NGC 2419. As extensively demonstrated in the literature (e.g. Dinescu et al. 2004; Massari et al. 2013; Sohn et al. 2013; Pryor et al. 2015), very distant objects such as quasars or background galaxies can be used to determine the absolute

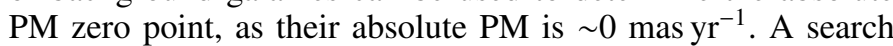
through the NED revealed no such objects in our FoV. However, when we inspected the images by eye to look for background galaxies, we found one object in both our HST and Gaia (which are the most shallow) data that has an extended structure typical of background galaxies, as shown in Fig. A.1. This is confirmed by the corresponding Gaia astrometric noise excess value which is larger than 10 . On the other hand, its overall profile is sufficiently point-like to be well described by the adopted PSF, as can be inferred from its QFIT value $<0.5$ (see Anderson \& King 2006). Moreover, it appears to be bright, with a signal-tonoise ratio $>150$ in all the HST exposures, and isolated, with no neighbouring sources affecting its centroid determination. This object thus has all the features required to provide a reliable determination of the absolute PM zero point. Its location in the Vector Point Diagram (VPD) with the corresponding uncertainties is shown in red in Fig. 1, and is centred on

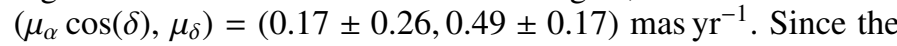
average PM of likely member stars (black points) is $\left(\mu_{\alpha} \cos (\delta)\right.$, $\left.\mu_{\delta}\right)=(0.001 \pm 0.007,-0.004 \pm 0.006) \mathrm{mas} \mathrm{yr}^{-1}$, that is, is consistent with zero within $1 \sigma$, the absolute PM of NGC 2419 is $\left(\mu_{\alpha} \cos (\delta), \mu_{\delta}\right)=(-0.17 \pm 0.26,-0.49 \pm 0.17) \operatorname{mas~yr}^{-1}$. In Galactic coordinates, this corresponds to $\left(\mu_{l} \cos b, \mu_{b}\right)=(0.43 \pm$ $0.09,-0.29 \pm 0.30)$ mas $\mathrm{yr}^{-1}$.

Given the large distance of NGC 2419, systematic uncertainties due to global systemic motions of the cluster such as expansion/contraction or rotation in the plane of the sky (see Massari et al. 2013) are negligible compared to the uncertainty on the absolute zero-point. For example, Baumgardt et al. (2009) found a rotation velocity of $3.1 \mathrm{~km} \mathrm{~s}^{-1}$ for NGC 2419 (with an rms of $4.0 \mathrm{~km} \mathrm{~s}^{-1}$ ), which translates into an additional systematic

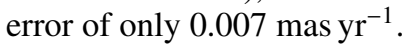

\section{The orbit of NGC 2419}

By combining the above measurements with the cluster's radial velocity $\left(v_{\mathrm{rad}}=-20.3 \pm 0.7 \mathrm{~km} \mathrm{~s}^{-1}\right.$, Baumgardt et al. 2009), distance $(87.5 \pm 3.3 \mathrm{kpc}$, Di Criscienzo et al. 2011b), and sky position $(\mathrm{RA}, \mathrm{Dec})=(114.535 \pm 0.004,+38.8824 \pm 0.0003) \mathrm{deg}$ (Goldsbury et al. 2010), we are able to determine, for the first time, the orbit of NGC 2419.

To this end, we transform these measurements to a heliocentric right-handed Cartesian reference frame, where $X$ points towards the Galactic centre, $Y$ in the direction of rotation, and $Z$ towards the Galactic North pole. This yields $(X, Y, Z)=(-79.1,-0.5,37.4)$ in $\mathrm{kpc}$, and $\left(V_{X}, V_{Y}, V_{Z}\right)=$ $(-32.6,-177.2,-119.3)$ in $\mathrm{km} \mathrm{s}^{-1}$. We then transform to a Galactocentric reference frame by assuming the the Sun's position and velocity to be $\left(X_{\odot}, Y_{\odot}, Z_{\odot}\right)=(-8.3,0,0.014) \mathrm{kpc}$, and $\left(V_{X, \odot}, V_{Y, \odot}, V_{Z, \odot}\right)=(11.1,240.24,7.25) \mathrm{km} \mathrm{s}^{-1}$ (see Schönrich et al. 2010).

We thus compute the orbit of NGC 2419 in a Galactic potential consisting of a flattened bulge, a gaseous exponential disc, thin and thick stellar exponential discs, and a flattened dark matter halo (for more details, see Piffl et al. 2014). The model has a total baryonic ( stars and cold gas) mass of $M_{\text {bary }}=5.3 \times 10^{10} M_{\odot}$ and a virial halo mass of $M_{200}=1.3 \times 10^{12} M_{\odot}$. The dark halo follows the Navarro et al. (1996) form, its flattening is $q=0.8$, and it has a concentration of $c_{200} \simeq 20$.

We use the phase-space position of the cluster we have just derived as the initial condition to integrate an orbit for approximately 4 Gyr forward and backward in time using an 8th order Runge-Kutta method. We also generate 100 realizations of the initial phase-space coordinates by assuming that the errors in the space of observables are Gaussian, and integrate them in the same way.

Figure 3 shows the trajectories on the sky of a subset of the orbits obtained in this way, that is, those with PM initial conditions within $1 \sigma$ of the measured values. The large errors on the measured PMs result in trajectories that cover a large portion of the sky. However more probable orbits typically do not deviate from the mean orbit shown by the dashed curve by more than a few degrees. Figure 3 also shows that this orbit is close to polar, indicating that most of the angular momentum is in the $Y$-direction. We find that the orbit rotates clockwise and has pericentre and apocentre distances $r_{\text {peri }}=53_{-26}^{+23} \mathrm{kpc}$, and $r_{\text {apo }}=98_{-1}^{+2} \mathrm{kpc}$, respectively.

Based on the position of NGC 2419 on the sky, Irwin (1999) suggested that it may have been a globular cluster associated with the dwarf spheroidal galaxy Sagittarius that was lost as soon as this entered the potential well of the Milky Way. Our PM measurements show that the sense of the rotation of Sagittarius and NGC2419 about the Galactic centre are the same, and therefore an association appears rather likely. Despite the fact that NGC 2419 lies at a much larger distance than the current orbit of Sagittarius (see e.g. Law \& Majewski 2010a, and the magenta line in Fig. 3), it must be borne in mind that if Sagittarius was much more massive in the past, its debris will be located at a large range of distances reflecting the initial energy spread 

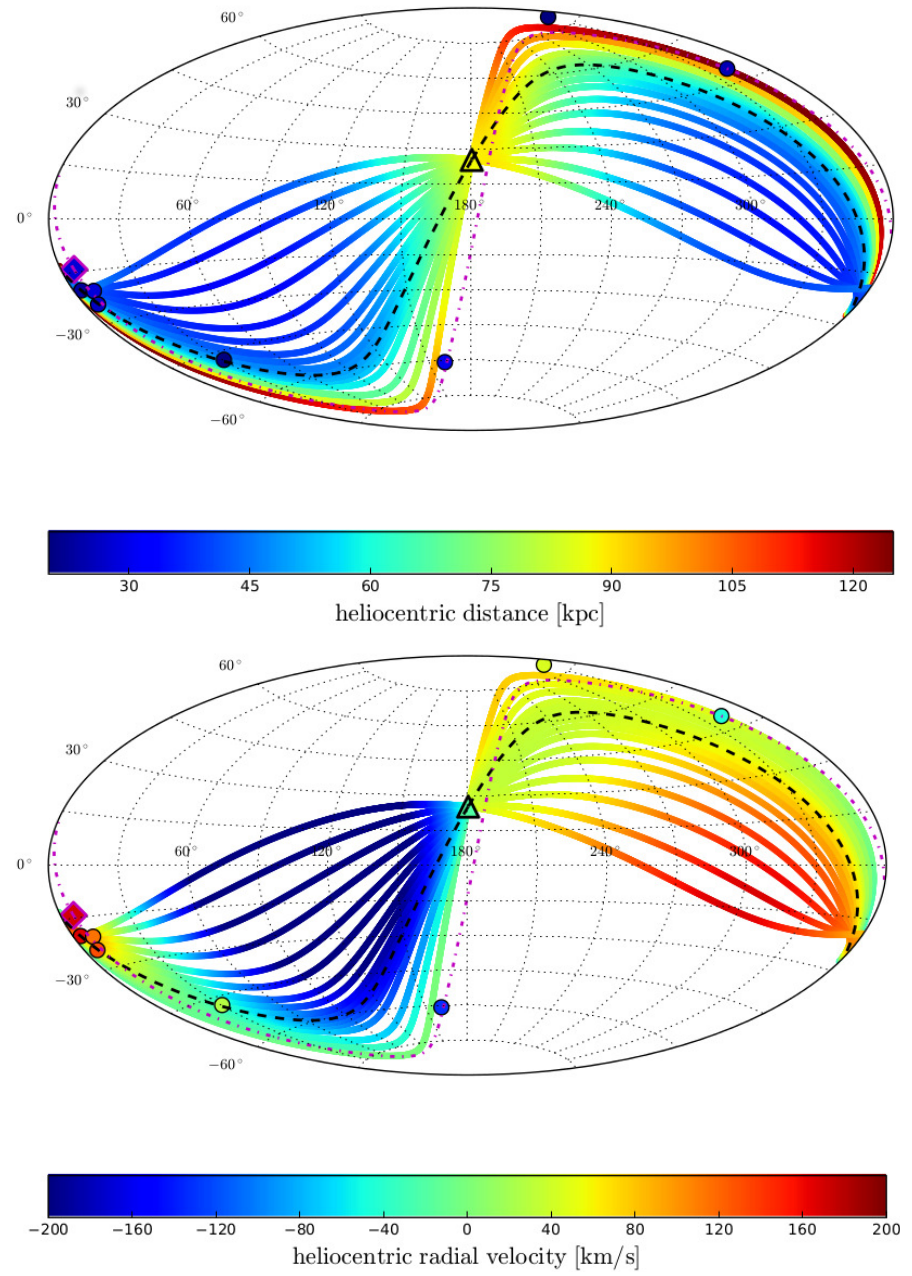

Fig. 3. Top panel: trajectory on the sky of NGC 2419's possible orbits (within $1 \sigma$ ), where that obtained by starting from its current position and velocity (indicated by a triangle) is shown with the black dashed line. The colour-coding represents the (heliocentric) distance. For comparison, we also show the current position and orbit of the Sagittarius dwarf spheroidal galaxy (diamond and magenta dot-dashed line), integrated in the same Galactic potential, and the positions of globular clusters (coloured circles) possibly associated to Sagittarius according to Law \& Majewski (2010b). Bottom panel: as the top panel, but where the colour-coding represents the (heliocentric) radial velocity.

(e.g. Helmi \& White 2001, predicted debris to lie at distances close to $100 \mathrm{kpc}$; see also Gibbons et al. 2014). Dynamical friction can also act in the same sense and make the orbit of Sagittarius sink towards the Galactic centre with time.

Furthermore, Belokurov et al. (2014) recently suggested that a tidal stream consisting of blue horizontal branch stars (reported first by Newberg et al. 2003) found to overlap with NGC 2419 spatially as well as in line-of-sight velocity is part of the trailing stream of Sagittarius. Although, for the streams, the original model of Law \& Majewski (2010a) predicted a different trend of line-of-sight velocity with angular phase than observed, the more recent model of Vera-Ciro \& Helmi (2013), in which the dark halo of the Galaxy is oblate near the centre and significantly triaxial at large distances (and which includes the gravitational effect of the Large Magellanic Could), fares better. Interestingly, the tangential velocity predicted by this model (see the righthand-side of Fig. 5 in Vera-Ciro \& Helmi 2013) is also in good agreement with what we have just derived for NGC 2419.

\section{Conclusions}

We have presented the first measurement of the proper motion of the intriguing globular cluster NGC 2419 thanks to the unique combination of HST and Gaia data. By using a background galaxy to tie our measurements to an absolute reference frame, we determined the absolute PM of NGC 2419 to be $\left(\mu_{\alpha} \cos (\delta)\right.$, $\left.\mu_{\delta}\right)=(-0.17 \pm 0.26,-0.49 \pm 0.17) \mathrm{mas} \mathrm{yr}^{-1}$.

Numerical integration of the possible orbits in a Galactic potential starting from the current location and velocity of NGC 2419 show the cluster to be on an elongated orbit with a pericentre at $\sim 53 \mathrm{kpc}$ and an apocentre at $\sim 98 \mathrm{kpc}$. Its orbit is close to polar and rotates in the same sense around the Milky Way as the Sagittarius dwarf galaxy. Our analysis suggests that it is very likely that NGC 2419 originated in the Sagittarius system. By combining all the information we have about Sagittarius, its streams and its likely former globular cluster NGC 2419, we may also be very close to pinning down the gravitational potential of the Milky Way at large radii, as well as reconstructing the remarkable history of the Sagittarius dwarf galaxy.

Acknowledgements. We thank the anonymous referee for comments and suggestions that improved the presentation of our results. D.M. and G.F. have been supported by the FIRB 2013 (MIUR grant RBFR13J716). A.H. and L.P. acknowledge financial support from a Vici grant from NWO. This work has made use of data from the European Space Agency (ESA) mission Gaia (http://www.cosmos.esa.int/gaia), processed by the Gaia Data Processing and Analysis Consortium (DPAC, http://www. cosmos.esa.int/web/gaia/dpac/consortium). Funding for the DPAC has been provided by national institutions, in particular the institutions participating in the Gaia Multilateral Agreement.

\section{References}

Anderson, J. 2007, Inst. Sci. Rep. ACS 2007-08

Anderson, J., \& Bedin, L. R. 2010, PASP, 122, 1035

Anderson, J., \& King, I. 2006, STScI Inst. Sci. Rep. ACS 2006-01 (Baltimore: STScI)

Baumgardt, H., Côté, P., Hilker, M., et al. 2009, MNRAS, 396, 2051

Bellini, A., Anderson, J., \& Bedin, L. R. 2011, PASP, 123, 622

Bellini, A., Anderson, J., van der Marel, R. P., et al. 2014, ApJ, 797, 115

Belokurov, V., Zucker, D. B., Evans, N. W., et al. 2006, ApJ, 642, L137

Belokurov, V., Koposov, S. E., Evans, N. W., et al. 2014, MNRAS, 437, 116

Cohen, J. G., Kirby, E. N., Simon, J. D., \& Geha, M. 2010, ApJ, 725, 288

di Criscienzo, M., D’Antona, F., Milone, A. P., et al. 2011a, MNRAS, 414, 3381

Di Criscienzo, M., Greco, C., Ripepi, V., et al. 2011b, AJ, 141, 81

Dinescu, D. I., Majewski, S. R., Girard, T. M., et al. 2002, ApJ, 575, L67

Dinescu, D. I., Keeney, B. A., Majewski, S. R., \& Girard, T. M. 2004, AJ, 128, 687

Gaia Collaboration (Brown, A. G. A., et al.) 2016, A\&A, 595, A2

Gibbons, S. L. J., Belokurov, V., \& Evans, N. W. 2014, MNRAS, 445, 3788

Goldsbury, R., Richer, H. B., Anderson, J., et al. 2010, AJ, 140, 1830

Harris, W. E. 1996, AJ, 112, 1487

Helmi, A., \& White, S. D. M. 2001, MNRAS, 323, 529

Helmi, A., Veljanoski, J., Breddels, M. A., Tian, H., \& Sales, L. V. 2017, A\&A, 598, A58

Irwin, M. 1999, IAU Symp., 192, 409

Law D. R., \& Majewski S. R. 2010a, ApJ, 714, 229

Law D. R., \& Majewski S. R. 2010b, ApJ, 718, 1128

Lee, Y.-W., Han, S.-I., Joo, S.-J., et al. 2013, ApJ, 778, L13

Lindegren, L., Lammers, U., Bastian, U., et al. 2016, A\&A, 595, A4

Massari, D., Bellini, A., Ferraro, F. R., et al. 2013, ApJ, 779, 81

Massari, D., Fiorentino, G., McConnachie, A., et al. 2016, A\&A, 595, L2

Mucciarelli, A., Bellazzini, M., Ibata, R., et al. 2012, MNRAS, 426, 2889

Navarro J. F., Frenk C. S., \& White S. D. M. 1996, ApJ, 462, 563

Newberg, H. J., Yanny, B., Grebel, E. K., et al. 2003, ApJ, 596, L191

Piffl, T., Binney, J., McMillan, P. J., et al. 2014, MNRAS, 445, 3133

Pryor, C., Piatek, S., \& Olszewski, E. W. 2015, AJ, 149, 42

Schönrich, R., Binney, J., \& Dehnen, W. 2010, MNRAS, 403, 1829

Sohn, S. T., Besla, G., van der Marel, R. P., et al. 2013, ApJ, 768, 139

Ubeda, L., \& Anderson, J. 2012, STScI Inst. Sci. Rep. ACS 2012-03 (Baltimore: $\mathrm{STScI})$

Vera-Ciro, C., \& Helmi, A. 2013, ApJ, 773, L4

Watkins, L. L., \& van der Marel, R. P. 2016, ApJ, submitted

[arXiv: 1611.03170] 
D. Massari et al.: The power of teaming up HST and Gaia: the first proper motion measurement of the distant cluster NGC 2419

\section{Appendix A: Additional figure}

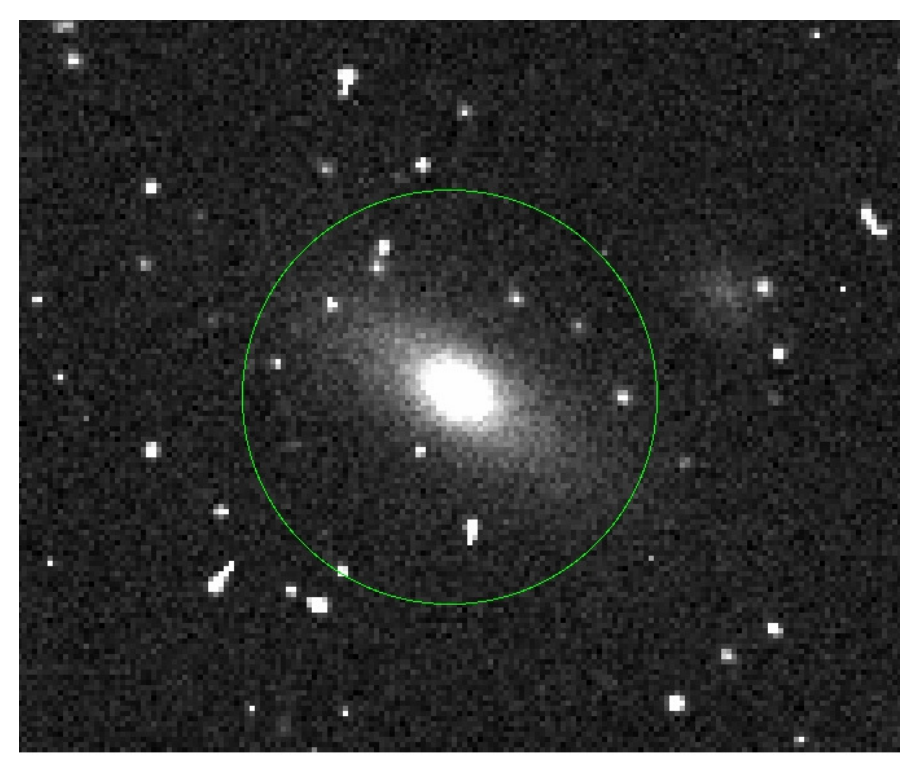

Fig. A.1. Background galaxy in one HST F606W exposure used to determine the absolute PM zero points. 